Y. WATANABE

KODAI MATH. J.

5 (1982), 111-121

\title{
RIEMANNIAN METRICS ON PRINCIPAL CIRCLE BUNDLES OVER LOCALLY SYMMETRIC KÄHLERIAN MANIFOLDS
}

\author{
By Yoshiyuki Watanabe
}

\section{Introduction.}

In Riemannian Geometry, one of the most interesting problems is to find all Einsteinian manifolds. A. Besse has suggested the research for Einsteinian manifolds satisfying the condition (see [3], p. 165)

$$
R_{\imath p q r} R_{j}^{p q r}=\text { constant } g_{\imath \jmath},
$$

where $g=\left(g_{i j}\right)$ is the Riemannian metric and $R=\left(R^{\imath}{ }_{j k l}\right)$ is the curvature tensor. Its typical examples are a locally symmetric spaces and a harmonic Riemannian manifold (cf. [13]). But the author [17] has recently shown that $S p(2) / S U(2)$ of Berger (cf. [2]) is an Einsteinian manifold satisfying (*).

On the other hand, J.E. D'Atri and H.K. Nickerson has initiated a study of the Riemannian manifold whose local geodesic symmetries are volume-preserving (up to sign). In this paper, we call such a manifold a (locally) volume symmetric space. It has been studied by J. E. D'Atri and H. K. Nickerson ([5]. [6]), K. Sekigawa ([15]) and Y. Watanabe ([16]). This class of manifolds obviously includes harmonic Riemannian manifolds and locally symmetric spaces. Then we are interested in Einsteinian manifolds (especially Einsteinian manifolds satisfying $(*))$, which are volume symmetric.

In this paper, we consider the Riemannian metric $\tilde{g}(t)$ given on a principal circle bundle $P$ over a Kählerian manifold $M$ (cf. $\S 2$ ). We show that if $M$ is locally symmetric, then $(P, \tilde{g}(t))$ is locally homogeneous and volume symmetric. Especially we also remark that an Einsteinian metric is given on $P$ in the case that $M$ is Einsteinian.

In $\S 2$, we define the Riemannian metric on a principal circle bundle over an $n$-dimensional Riemannian manifold and give the fundamental formulas. In $\S 3$, we calculate the covariant derivative of the Riemannian curvature tensor by using the structure equations obtained in $\S 2$. In $\S 4$, we define a tensor field $T$ of type $(1,2)$ on $P$ and state its properties for later use. In $\S 5, M$ is assumed to be a locally symmetric Kählerian manifold. Then by using the equations obtained in sections 3 and 4 , we show the main results. In the last section, an

Received September 20, 1980. 
example is stated in detail, because in the opinion of the author the method in this paper should have other applications.

The author wishes to express his sincere thanks to Prof. S. Ishihara, Prof. S. Tachibana and Prof. K. Takamatsu for their valuable suggestions and encouragements.

\section{A principal circle bundle.}

Let $\tilde{M}$ and $M$ be two Riemannian manifolds of dimensions $m$ and $n$ respectively, where $m-n>0$. A Riemannian submersions, which shall call simply submersion, is a differentiable map $\pi: \tilde{M} \rightarrow M$, which is onto, and for which at each point $x \in M$ the differential map $\pi_{*}$ acts as an orthogonal projection of $T \tilde{x} \tilde{M}$ onto $T_{x} M$, where $x=\pi(\tilde{x})$. Then for each point $x \in M$ its inverse image $\pi^{-1}(x)$ is a submanifold of $M$ of dimension $m-n$, which is called the fibre over $x$.

A vector field on $\tilde{M}$ is called vertical if it is always tangent to fibres and holizontal if always orthogonal to fibres; we use corresponding terminology for individual tangent vectors. We denote the Riemannian connections by $\tilde{\nabla}$ on $\tilde{M}$, $\nabla$ on $M$ and $\bar{\nabla}$ on the fibres, defined by the Riemannian metrics $\tilde{g}$ on $\tilde{M}, g$ on $M$ and $\bar{g}$ on the fibres respectively. For a submersion $\pi: \tilde{M} \rightarrow M$, let $\mathscr{H}$ and $\mathscr{V}$ denote the projections of the tangent space of $\tilde{M}$ onto the subspaces of horizontal and vertical vectors, respectively. We define a vector field $\tilde{X}$ on $\tilde{M}$ to be basic provided $\tilde{X}$ is horizontal and $\pi$-related to a vector field $X$ on $M$. Every vector field $X$ on $M$ has a unique horizontal lift $\tilde{X}$ to $\tilde{M}$, and $\tilde{X}$ is basic. Thus $\tilde{X} \leftrightarrow X$ is a one-to-one correspondence between basic vector fields on $\tilde{M}$ and arbitrary vector fields on $M$. This correspondence preserves brackets, inner products and covariant derivatives to the following (see [12]).

LEMMA 2.1. If $X$ and $Y$ are basic vector fields on $M$, then

(1) $\langle\tilde{X}, \tilde{Y}\rangle=\langle X, Y\rangle \circ \pi$,

(2) $[\tilde{X}, \tilde{Y}]$ is the basic vector field corresponding to $[X, Y]$,

(3) $\tilde{\nabla}_{\tilde{X}} \tilde{Y}$ is the basic vector field corresponding to $\nabla_{X} Y$, where $\langle\tilde{X}, \tilde{Y}\rangle$ (resp. $\langle X, Y\rangle)$ is the inner product $\tilde{g}(\tilde{X}, \tilde{Y})$ (resp. $g(X, Y)$ ) of vectors $\tilde{X}$ and $\tilde{Y}$ of $\tilde{M}$ (resp. $X$ and $Y$ of $M$ ).

Let $P$ be a principal circle bundle over an $n$-dimensional manifold with projection $\pi, g$ a Riemannian metric on $M$ and $\eta$ a connection form on $P$ defining a connection in the bundle $P$. Functions on $M$ such as components of tensor fields on $M$ are considered sometimes as functions on $P$ in a natural way without any change of notations. We shall also agree on that indices $i, j, k$ and $l$ run from 1 to $n$ and $\alpha, \beta, \gamma$ and $\delta$ run from 0 to $n$. Since the structure group $S^{1}$ of $P$ is abelian, the structure equation is given by

$$
d \eta=\Omega
$$

where $\Omega$ is the curvature form of $\eta$. Then $\tilde{g}=\pi^{*} g+t^{2} \eta \otimes \eta$ for any $t>0$, is a 
Riemannian metric on $P$ with respect to which $\pi$ becomes a Riemannian submersion ([7], [12]). Explicitly if $\tilde{x} \in P, \tilde{X}, \tilde{Y} \in T \tilde{x} P$, then

$$
\tilde{g}(\tilde{X}, \tilde{Y})=g\left(\pi_{*} \tilde{X}, \pi_{*} \tilde{Y}\right)+t^{2} \eta(\tilde{X}) \eta(\tilde{Y}) .
$$

The action of $S^{1}$ on $P$ allows us to take a unit fundamental vector field $\xi$ such that

$$
\tilde{g}(\tilde{X}, \xi)=\operatorname{t\eta }(\tilde{X})
$$

for any vector field $\tilde{X}$ on $P$. If $\tilde{X}$ is basic, then $[\tilde{X}, \xi]$ is vertical. Thus since $[\tilde{X}, \xi]$ is horizontal (cf. [11]), we have the following

LEMMA 2.2 (cf. [7]). If $\tilde{X}$ is a basic vector field on $M$, then

$$
[\tilde{X}, \xi]=0 .
$$

\section{Structure equations of the fibring}

To calculate the Riemannian curvature tensor $\widetilde{R}$ of $\tilde{g}$ at any fixed point $\tilde{x} \in P$, we shall take a special orthonormal frame field on a neighborhood of $x=\pi(\tilde{x})$ such that $\nabla_{X_{i}} X_{j}=0$ at $x$ for any $i$ and $\jmath$. The basic vector fields $\tilde{X}_{i}$ corresponding to $X_{\imath}$ are orthonormal vector fields such that $\tilde{\nabla}_{\tilde{X}_{i}} \tilde{X}_{j}$ is vertical on the fibre passing through $\tilde{x}$ for any $i$ and $j$. Therefore we have a local orthonormal frame field $\left\{\xi, \tilde{X}_{1}, \cdots, \tilde{X}_{n}\right\}$ around $\tilde{x}$. Using the standard formula

$$
\begin{aligned}
2\left\langle\tilde{\nabla}_{\tilde{X}} \tilde{Y}, \tilde{Z}\right\rangle= & \tilde{X}\langle\tilde{Y}, \tilde{Z}\rangle+\tilde{Y}\langle\tilde{Z}, \tilde{Y}\rangle-\tilde{Z}\langle\tilde{X}, \tilde{Y}\rangle-\langle\tilde{X},[\tilde{Y}, \tilde{Z}]\rangle \\
& +\langle\tilde{Y},[\tilde{Z}, \tilde{X}]\rangle+\langle\tilde{Z},[\tilde{X}, \tilde{Y}]\rangle,
\end{aligned}
$$

for any vector fields $\tilde{X}, \tilde{Y}$ and $\tilde{Z}$ and the definition (2.2), we can prove the following lemma (cf. [14]).

LEMMA 3.1. The components of the connection $\tilde{\nabla}$ with respect to the orthonormal frame field taken above are given by

(1) $\tilde{\nabla}_{\xi} \xi=0$,

(2) $\tilde{\nabla}_{\xi} \tilde{X}_{\imath}=\nabla_{\tilde{X}_{i}} \xi=t \Sigma \Omega_{\imath \jmath} \tilde{X}_{\jmath}$,

(3) $\propto \mathcal{V}\left(\tilde{\nabla}_{\tilde{X}_{i}} \tilde{X}_{j}\right)=(1 / 2) \propto v\left[\tilde{X}_{\imath}, \tilde{X}_{\jmath}\right]=-t \Omega_{\imath \jmath} \xi$,

(4) $\mathscr{H}\left(\tilde{\nabla}_{\tilde{X}_{i}} \tilde{X}_{j}\right)=\Sigma S_{\imath j k} \tilde{X}_{k}$,

(5) $\left(\tilde{\nabla}_{\xi} \Omega\right)\left(\tilde{X}_{\imath}, \tilde{X}_{j}\right)=0$,

in a neighborhood of $\tilde{x}$, where $\Omega_{\imath \jmath}=\Omega\left(\tilde{X}_{\imath}, \tilde{X}_{\jmath}\right)$ and $S_{\imath \jmath k}=\left\langle\nabla_{X_{i}} X_{\jmath}, X_{k}\right\rangle \circ \pi$.

Let $\tilde{R}$ (resp. $R$ ) be the curvature tensor of $P($ resp. $M)$. Then by repeated use of Lemma 3.1, we can obtain the following 
LEMMA 3.2. (cf. [10]). The components of the curvature tensor $R$ with respect to the orthonormal frame $\left\{\xi, \tilde{X}_{1}, \cdots, \tilde{X}_{n}\right\}$ are given by

(1) $\tilde{R}_{\imath 0 \jmath 0}=t^{2} \Sigma \Omega_{j l} \Omega_{l \imath}$,

(2) $\tilde{R}_{\imath o j k}=\tilde{\nabla}_{\imath} \Omega_{j k}$,

(3) $\quad \tilde{R}_{\imath j k l}=R_{\imath j k l}-t^{2}\left(\Omega_{i l} \Omega_{j k}-\Omega_{\imath k} \Omega_{j l}-2 \Omega_{\imath j} \Omega_{k l}\right)$,

where $\tilde{R}_{\imath o \jmath 0}=\left\langle\tilde{R}\left(\tilde{X}_{\imath}, \xi\right) \tilde{X}_{\jmath}, \xi\right\rangle, \tilde{R}_{\imath o j k}=\left\langle\tilde{R}\left(\tilde{X}_{\imath}, \xi\right) \tilde{X}_{\jmath}, \tilde{X}_{k}\right\rangle, \tilde{R}_{\imath j k l}=\left\langle\tilde{R}_{(}\left(\tilde{X}_{\imath}, \tilde{X}_{j}\right) \tilde{X}_{k}, \tilde{X}_{l}\right\rangle$, $R_{\imath j k l}=\left\langle R\left(X_{\imath}, X_{j}\right) X_{k}, X_{l}\right\rangle$ and $\tilde{\nabla}_{\imath} \Omega_{j k}=\left(\tilde{\nabla}_{\tilde{X}_{i}} \Omega\right)\left(\tilde{X}_{\jmath}, \tilde{X}_{k}\right)$.

LEMMA 3.3. The curvature tensor $\widetilde{R}$ is expressed in the terms of $R_{\imath j k l}$ and $\Omega_{\imath}$ as follows:

(1) $\tilde{R}\left(\xi, \tilde{X}_{\imath}\right) \xi=t^{2} \Sigma \Omega_{j k} \Omega_{k \imath} \tilde{X}_{\jmath}$,

(2) $\tilde{R}\left(\xi, \tilde{X}_{\imath}\right) \tilde{X}_{j}=-t^{2} \Sigma\left(\Omega_{\imath k} \Omega_{k j} \xi-t \tilde{\nabla}_{\imath} \Omega_{j k} \tilde{X}_{k}\right)$,

(3) $\tilde{R}\left(\tilde{X}_{\imath}, \tilde{X}_{j}\right) \xi=t \Sigma \tilde{\nabla}_{k} \Omega_{\imath}, \tilde{X}_{k}$,

(4) $\tilde{R}\left(\tilde{X}_{\imath}, \tilde{X}_{j}\right) \tilde{X}_{k}=t \tilde{\nabla}_{k} \Omega_{\imath j} \xi+\Sigma\left[R_{\imath j k l}-t^{2}\left(\Omega_{i l} \Omega_{j k}-\Omega_{\imath k} \Omega_{j l}-2 \Omega_{\imath j} \Omega_{k l}\right)\right] X_{l}$.

We shall calculate the covariant derivative $\tilde{\nabla} \tilde{R}$ of the curvature tensor $\tilde{R}$ by using Lemmas 3.1, 3.2 and 3.3.

LEMMA 3.4 .

(1) $\left(\tilde{\nabla}_{\xi} \tilde{R}\right)\left(\xi, \tilde{X}_{\imath}\right) \xi=0$,

(2) $\left(\tilde{\nabla}_{\tilde{X}_{i}} \tilde{R}\right)\left(\xi, \tilde{X}_{j}\right) \xi=t^{2} \Sigma\left[\Omega_{k l} \Omega_{l j}\left(S_{\imath k a}+S_{\imath a k}\right)\right.$

$\left.+S_{i l k}\left(\Omega_{k j} \Omega_{a l}+\Omega_{a k} \Omega_{l j}\right)+\Omega_{l j} \tilde{\nabla}_{\imath} \Omega_{a l}+\Omega_{a l} \tilde{\nabla}_{\imath} \Omega_{l \jmath}\right] \tilde{X}_{a}$,

(3) $\left(\tilde{\nabla}_{\xi} \tilde{R}\right)\left(\xi, \tilde{X}_{\imath}\right) \tilde{X}_{\jmath}=-t \Sigma\left[\xi\left(\nabla_{\imath} \Omega_{j k}\right)-t \Omega_{l k} \tilde{\nabla}_{\imath} \Omega_{j l}+\Omega_{i l} \tilde{\nabla}_{l} \Omega_{j k}+\Omega_{j l} \tilde{\nabla}_{\imath} \Omega_{l k}\right] \tilde{X}_{k}$,

(4) $\left(\tilde{\nabla}_{\tilde{X}_{k}} \tilde{R}\right)\left(\xi, \tilde{X}_{\imath}\right) \tilde{X}_{j}=-\Sigma\left[t^{2}\left\{\left(\tilde{\nabla}_{k} \Omega_{j l}\right) \Omega_{l i}+\Omega_{j l}\left(\tilde{\nabla}_{k} \Omega_{l \imath}\right)\right\} \xi\right.$

$\left.-t \Omega_{k l} R\left(\tilde{X}_{l}, \tilde{X}_{\imath}\right) \tilde{X}_{j}-S_{k i l} \tilde{R}\left(\xi, \tilde{X}_{l}\right) \tilde{X}_{j}-S_{k j l} \tilde{R}\left(\xi, \tilde{X}_{\imath}\right) \tilde{X}_{l}\right]$,

(5) $\left(\tilde{\nabla}_{\tilde{X}_{k}} \tilde{R}\right)\left(\tilde{X}_{\imath}, \tilde{X}_{j}\right) \xi=\Sigma\left[t \tilde{X}_{k}\left(\tilde{\nabla}_{l} \Omega_{\imath j}\right) \tilde{X}_{l}+t S_{k l a} \tilde{\nabla}_{l} \Omega_{\imath \jmath} \tilde{X}_{a}-t \Omega_{k l} \tilde{R}\left(\tilde{X}_{\imath}, \tilde{X}_{j}\right) \tilde{X}_{l}\right.$

$-S_{k i l} \tilde{R}\left(\tilde{X}_{l}, \tilde{X}_{\jmath}\right) \xi-t S_{k j l} \tilde{\nabla}_{a} \Omega_{i l} \tilde{X}_{a}+t^{3} \Omega_{a l}\left(\Omega_{k \imath} \Omega_{l j}-\Omega_{k j} \Omega_{l \imath}\right) \tilde{X}_{a}+t \tilde{\nabla}_{k} \Omega_{\imath \jmath} \xi$,

(6) $\left(\tilde{\nabla}_{\tilde{X}_{h}} \tilde{R}\right)\left(\tilde{X}_{\imath}, \tilde{X}_{j}\right) \tilde{X}_{k}=\Sigma\left[\left\{\left\langle\left(\nabla_{X_{h}} R\right)\left(X_{\imath}, X_{j}\right) X_{k}, X_{l}\right\rangle-t^{2}\left(\Omega_{j k} \tilde{\nabla}_{h} \Omega_{i l}\right.\right.\right.$

$\left.\left.+\Omega_{i l} \tilde{\nabla}_{h} \Omega_{j k}-\Omega_{j l} \tilde{\nabla}_{h} \Omega_{\imath k}-\Omega_{\imath k} \tilde{\nabla}_{h} \Omega_{j l}-2 \Omega_{k l} \tilde{\nabla}_{h} \Omega_{\imath j}-2 \Omega_{\imath j} \tilde{\nabla}_{h} \Omega_{k l}\right)\right\} X_{l}$

$-t \Omega_{h l} \tilde{R}_{\imath j k l} \xi+S_{h l a} \tilde{R}_{\imath j k l} \tilde{X}_{a}-S_{h i l} \tilde{R}\left(\tilde{X}_{l}, \tilde{X}_{j}\right) \tilde{X}_{k}-S_{h j l} \tilde{R}\left(X_{\imath}, \tilde{X}_{l}\right) \tilde{X}_{k}$

$-S_{\imath k l} \tilde{R}\left(\tilde{X}_{\imath}, \tilde{X}_{j}\right) \tilde{X}_{l}+t^{2}\left(-t \Omega_{k l} \Omega_{l j} \xi+\tilde{\nabla}_{\imath} \Omega_{h l} \tilde{X}_{l}\right) \Omega_{h i}+t^{2} \Omega_{h k}\left(\tilde{\nabla}_{l} \Omega_{\imath j}\right) \tilde{X}_{l}$

$\left.+t^{2}\left(t \Omega_{k l} \Omega_{l i} \xi+\tilde{\nabla}_{\imath} \Omega_{k l} \tilde{X}_{l}\right) \Omega_{h \jmath}\right]$. 
Proof. Using the standard formula

$$
\begin{aligned}
\left(\tilde{\nabla}_{\tilde{X}} \tilde{R}\right)(\tilde{Y}, \tilde{Z}) \widetilde{W}= & \tilde{\nabla}_{\tilde{X}}(\tilde{R}(\tilde{Y}, \tilde{Z}) \widetilde{W})-\tilde{R}\left(\tilde{\nabla}_{\tilde{X}} \tilde{Y}, \tilde{Z}\right) \widetilde{W} \\
& -\tilde{R}\left(\tilde{Y}, \tilde{\nabla}_{\tilde{X}} \tilde{Z}\right) \widetilde{W}-\tilde{R}(\tilde{Y}, \tilde{Z})\left(\tilde{\nabla}_{\tilde{X}} \widetilde{W}\right),
\end{aligned}
$$

for any vector fields $\tilde{X}, \tilde{Y}$ and $\tilde{Z}$, we shall prove this lemma. By using the above formula for $X=\xi, Y=\xi, Z=X_{\imath}$ and $W=\xi$, we have, from (1) and (2) of Lemma 3.1 and (1) of Lemma 3.2,

$$
\begin{aligned}
\left(\tilde{\nabla}_{\xi} \tilde{R}\right)\left(\xi, \tilde{X}_{\imath}\right) \xi & =\tilde{\nabla}_{\xi}\left(\tilde{R}_{(\xi,}\left(\tilde{X}_{\imath}\right) \xi\right)-\tilde{R}\left(\tilde{\nabla}_{\xi} \xi, \tilde{X}_{\imath}\right) \xi-\tilde{R}\left(\xi, \tilde{\nabla}_{\xi} \tilde{X}_{\imath}\right) \xi-\tilde{R}\left(\xi, \tilde{X}_{\imath}\right)\left(\tilde{\nabla}_{\xi} \xi\right) \\
& =\Sigma\left[t^{2} \tilde{\nabla}_{\xi}\left(\Omega_{j l} \Omega_{\iota \imath} \tilde{X}_{j}\right)-t \Omega_{i l} \tilde{R}\left(\xi, \tilde{X}_{l}\right) \xi\right] \\
& =\Sigma\left[t^{2}\left(\Omega_{j l} \Omega_{l \imath}\right) \tilde{\nabla}_{\xi} \tilde{X}_{j}-t^{2} \Omega_{i l} \Omega_{j k} \Omega_{k l} \tilde{X}_{\jmath}\right]=0 .
\end{aligned}
$$

Thus we obtain the formula (1). Similarly, we can obtain the formulas (2), (3), (4), (5) and (6) by Lemmas 3.1 and 3.3.

\section{The tensor field $T$ of type $(1,2)$ on $P$.}

We shall give a tensor field $T$ of type $(1,2)$, which plays an important role in our assertion, and study its properties. Let us define $T$ by

$$
T(\tilde{X}, \tilde{Y})=t[\Omega(\tilde{X}, \tilde{Y}) \xi+t(\eta(\tilde{X}) \phi \tilde{Y}-\eta(\tilde{Y}) \phi \tilde{X})],
$$

for any vector fields $\tilde{X}$, $\tilde{Y}$ on $P$, where $\phi$ is defined by $\tilde{g}(\phi \tilde{X}, \tilde{Y})=\Omega(\tilde{X}, \tilde{Y})$, then $T$ is a tensor field of type $(1,2)$ on $P$ such that $T(\tilde{X}, \tilde{Y})=-T(\tilde{Y}, \tilde{X})$. With respect to the orthonormal frame field taken in section 3 , we have

$$
T\left(\xi, \tilde{X}_{\imath}\right)=t \Sigma \Omega_{\imath \imath} \tilde{X}_{l}
$$

and

$$
T\left(\tilde{X}_{\imath}, \tilde{X}_{\jmath}\right)=t \Omega_{\imath} \xi
$$

Hence we obtain

LEMMA 4.1.

(1) $\left(\tilde{\nabla}_{\xi} T\right)\left(\tilde{X}_{\imath}, \tilde{X}_{\jmath}\right)=\left(\tilde{\nabla}_{\xi} T\right)\left(\xi, \tilde{X}_{\imath}\right)=0$,

(2) $\left(\tilde{\nabla}_{\tilde{X}_{i}} T\right)\left(\xi, \tilde{X}_{j}\right)=t \Sigma\left(\tilde{\nabla}_{\imath} \Omega_{\jmath k}\right) \tilde{X}_{k}$,

(3) $\left(\tilde{\nabla}_{\tilde{X}_{i}} T\right)\left(\tilde{X}_{\jmath}, \tilde{X}_{k}\right)=t\left(\tilde{\nabla}_{\imath} \Omega_{\jmath k}\right)+\Sigma t^{2}\left(\Omega_{j k} \Omega_{\imath l}+\Omega_{\imath j} \Omega_{k l}-\Omega_{\imath k} \Omega_{\jmath l}\right) \tilde{X}_{l}$.

Proof. Using the standard formula

$$
\left(\tilde{\nabla}_{\tilde{X}} T\right)(\tilde{Y}, \tilde{Z})=\tilde{\nabla}_{\tilde{X}}(T(\tilde{Y}, \tilde{Z}))-T\left(\tilde{\nabla}_{\tilde{X}} \tilde{Y}, \tilde{Z}\right)-T\left(\tilde{Y}, \tilde{\nabla}_{\tilde{X}} \tilde{Z}\right),
$$

for any vector fields $\tilde{X}, \tilde{Y}$ and $\tilde{Z}$, we shall prove this lemma. Using the above 
formula for $\tilde{X}=\tilde{X}_{\imath}, \tilde{Y}=\tilde{X}_{\text {, and }} \tilde{Z}=\tilde{X}_{k}$, we verify the third assertion as follows: By (2) and (3) of Lemma 3.1 and the definition (4.1) of $T$, we have

$$
\begin{aligned}
\left(\tilde{\nabla}_{\tilde{X}_{\imath}} T\right)\left(\tilde{X}_{\imath}, \tilde{X}_{k}\right)= & \tilde{\nabla}_{\tilde{X}_{i}}\left(T\left(\tilde{X}_{\jmath}, \tilde{X}_{k}\right)\right)-T\left(\tilde{\nabla}_{\tilde{X}_{i}} \tilde{X}_{\jmath}, \tilde{X}_{k}\right)-T\left(\tilde{X}_{\jmath}, \tilde{\nabla}_{\tilde{X}_{i}} \tilde{X}_{k}\right) \\
= & \tilde{\nabla}_{\tilde{X}_{i}}\left[t \Omega\left(\tilde{X}_{\jmath}, \tilde{X}_{k}\right) \xi+t^{2}\left(\eta\left(\tilde{X}_{j}\right) \phi \tilde{X}_{k}-\eta\left(\tilde{X}_{k}\right) \phi \tilde{X}_{\jmath}\right)\right] \\
& -\left[t \Omega\left(\tilde{\nabla}_{\tilde{X}_{i}} \tilde{X}_{\jmath}, \tilde{X}_{k}\right) \xi+t^{2}\left(\eta\left(\tilde{\nabla}_{\tilde{X}_{i}} \tilde{X}_{j}\right) \phi \tilde{X}_{k}-\eta\left(\tilde{X}_{k}\right) \phi \tilde{X}_{j}\right)\right] \\
& -\left[t \Omega\left(\tilde{X}_{\jmath}, \tilde{\nabla}_{\tilde{X}_{i}} \tilde{X}_{k}\right) \xi+t^{2}\left(\eta\left(\tilde{X}_{j}\right) \phi\left(\tilde{\nabla}_{\tilde{X}_{i}} \tilde{X}_{k}\right)-\eta\left(\tilde{\nabla}_{\tilde{X}_{i}} \tilde{X}_{j}\right) \phi \tilde{X}_{\jmath}\right]\right. \\
= & t\left(\tilde{\nabla}_{\imath} \Omega_{j k}\right) \xi+t^{2} \sum\left(\Omega_{j k} \Omega_{i l}+\Omega_{\imath j} \Omega_{k l}-\Omega_{\imath k} \Omega_{j l}\right) \tilde{X}_{l} .
\end{aligned}
$$

The assertions (1) and (2) follow similarly from (4.1), (4.2), (4.3) and Lemma 3.1.

Here if we put

$$
\left(T_{\tilde{X}} \cdot T\right)(\tilde{Y}, \tilde{Z})=T(\tilde{X}, T(\tilde{Y}, \tilde{Z}))-T(T(\tilde{X}, \tilde{Y}), \tilde{Z})-T(\tilde{Y}, T(\tilde{X}, \tilde{Z})),
$$

for any vector fields $\tilde{X}, \tilde{Y}$ and $\tilde{Z}$, the $T_{\tilde{X}} \cdot T$ is a tensor field of type $(1,2)$ on $P$ and satisfies the following

LEMMA 4.2.

(1) $\left(T_{\tilde{X}_{\imath}} \cdot T\right)\left(\tilde{X}_{\imath}, \tilde{X}_{j}\right)=-t^{2} \Sigma\left(\Omega_{j k} \Omega_{i l}+\Omega_{\imath j} \Omega_{k l}-\Omega_{\imath k} \Omega_{j l}\right) \tilde{X}_{l}$,

(2) $\left(T_{\tilde{X}_{\imath}} \cdot T\right)\left(\xi, \tilde{X}_{j}\right)=\left(T_{\xi} \cdot T\right)\left(\tilde{X}_{\imath}, \tilde{X}_{j}\right)=0$.

Proof. By (4.1), (4.2), (4.3) and Lemma 3.1, we have

$$
\begin{aligned}
& T\left(\tilde{X}_{\imath}, T\left(\tilde{X}_{\jmath}, \tilde{X}_{k}\right)\right)-T\left(T\left(\tilde{X}_{\imath}, \tilde{X}_{\jmath}\right), \tilde{X}_{k}\right)-T\left(\tilde{X}_{\jmath}, T\left(\tilde{X}_{\imath}, \tilde{X}_{k}\right)\right) \\
& \quad=t\left[\Omega_{j k} T\left(\tilde{X}_{\imath}, \xi\right)-\Omega_{\imath \jmath} T\left(\xi, \tilde{X}_{k}\right)-\Omega_{\imath k} T\left(\tilde{X}_{\jmath}, \xi\right)\right] \\
& \quad=-t^{2} \Sigma\left(\Omega_{j k} \Omega_{i l}+\Omega_{\imath j} \Omega_{k l}-\Omega_{\imath k} \Omega_{j l}\right) \tilde{X}_{l} .
\end{aligned}
$$

Similarly we can verify the assertion (2).

Now for later use, we define a tensor field $T_{\tilde{X}} \cdot \tilde{R}$ of type $(1,3)$ on $P$ by

$$
\begin{aligned}
\left(T_{\tilde{W}} \cdot \tilde{R}\right)(\tilde{X}, \tilde{Y}) \tilde{Z}= & T(\widetilde{W}, \tilde{R}(\tilde{X}, \tilde{Y}) \tilde{Z})-\tilde{R}(T(\widetilde{W}, \tilde{X}), \tilde{Y}) \tilde{Z} \\
& -\tilde{R}(\tilde{X}, T(\widetilde{W}, \tilde{Y})) \tilde{Z}-\tilde{R}(\tilde{X}, \tilde{Y})(T(\widetilde{W}, \tilde{Z})),
\end{aligned}
$$

for any vector fields $\tilde{X}, \tilde{Y}, \tilde{Z}$ and $\widetilde{W}$. Then we have the following

LEMMA 4.3.

(1) $\left(T_{\xi} \cdot \widetilde{R}\right)\left(\xi, \tilde{X}_{\imath}\right)=0$ ，

(2) $\left(T_{\tilde{X}_{i}} \cdot \tilde{R}\right)\left(\xi, \tilde{X}_{j}\right) \xi=-t^{2} \sum \Omega_{\imath k}\left(\tilde{\nabla}_{j} \Omega_{k l}\right) \tilde{X}_{l}$,

(3) $\left(T_{\xi} \cdot \tilde{R}\right)\left(\xi, \tilde{X}_{\imath}\right) \tilde{X}_{\jmath}=t^{2} \Sigma\left[-\Omega_{k l}\left(\tilde{\nabla}_{\imath} \Omega_{j k}\right)+\Omega_{\imath k}\left(\tilde{\nabla}_{k} \Omega_{\jmath l}\right)+\Omega_{j k}\left(\tilde{\nabla}_{\imath} \Omega_{k l}\right)\right] \tilde{X}_{l}$, 
(4) $\quad\left(T_{\tilde{X}_{k}} \cdot \tilde{R}\right)\left(\xi, \tilde{X}_{\imath}\right) \tilde{X}_{\jmath}=\Sigma\left[t^{3} \Omega_{j l} \Omega_{l \imath} \Omega_{k a} \tilde{X}_{a}-t^{2} \Omega_{k l}\left(\tilde{\nabla}_{\imath} \Omega_{\jmath l}\right) \xi\right.$

$$
\left.+t \Omega_{k l} \tilde{R}\left(\tilde{X}_{l}, \tilde{X}_{\imath}\right) \tilde{X}_{\jmath}-t^{3} \Omega_{k j} \Omega_{a l} \Omega_{l \imath} \tilde{X}_{a}\right],
$$

(5) $\quad\left(T_{\tilde{X}_{n}} \cdot \tilde{R}\right)\left(\tilde{X}_{\imath}, \tilde{X}_{j}\right) \xi=\Sigma\left[t \Omega_{k l}\left(\tilde{\nabla}_{l} \Omega_{\imath \jmath}\right) \xi-t^{3}\left(\Omega_{k \imath} \Omega_{a l} \Omega_{l j}\right) \tilde{X}_{a}\right.$

$$
\left.+t^{2} \Omega_{k j} \Omega_{a l} \Omega_{l \imath} \tilde{X}_{a}+t \Omega_{k l} \tilde{R}\left(\tilde{X}_{\imath}, \tilde{X}_{\jmath}\right) \tilde{X}_{l}\right],
$$

(6) $\left(T_{\tilde{X}_{h}} \cdot \tilde{R}\right)\left(\tilde{X}_{\imath}, \tilde{X}_{j}\right) \tilde{X}_{k}=\Sigma\left[-t \Omega_{h l} \tilde{\nabla}_{k} \Omega_{\imath \jmath} \tilde{X}_{l}+t \Omega_{k l} \tilde{R}_{\imath \jmath k l} \xi+t^{3} \Omega_{h \imath} \Omega_{k l} \Omega_{l j} \xi\right.$

$$
\left.+t^{2} \Omega_{h i}\left(\tilde{\nabla}_{\jmath} \Omega_{k l}\right) \tilde{X}_{l}-t^{3} \Omega_{h j} \Omega_{k l} \Omega_{l \imath} \xi-t^{2} \Omega_{h k}\left(\tilde{\nabla}_{l} \Omega_{\imath \jmath}\right) \tilde{X}_{l}\right] \text {. }
$$

Proof. By Lemmas 3.1 and 3.2, (2.4), (4.1), (4.2), (4.3) and (4.6) imply that

$$
\begin{aligned}
(T \xi \cdot \tilde{R})\left(\xi, \tilde{X}_{\imath}\right) \xi & =T\left(\xi, \tilde{R}\left(\xi, \tilde{X}_{\imath}\right) \xi\right)-\tilde{R}\left(T(\xi, \xi), \tilde{X}_{\imath}\right) \xi-\tilde{R}\left(\xi, T\left(\xi, \tilde{X}_{\imath}\right)\right) \xi-\tilde{R}\left(\xi, \tilde{X}_{\imath}\right) T(\xi, \xi) \\
& =\Sigma\left[t^{2} \Omega_{j l} \Omega_{l \imath} T\left(\xi, \tilde{X}_{j}\right)-t \Omega_{i l} \tilde{R}\left(\xi, \tilde{X}_{l}\right) \xi\right]=0 .
\end{aligned}
$$

Similarly we can obtain the equations (2), (3), (4), (5) and (6).

\section{Main results.}

Let $M$ be a locally symmetric Kählerian manifold with a Kählerian metric $g$ and the fundamental 2-form $\Theta$. Let $P$ be a principal circle bundle over $M, \tilde{g}$ a Riemannian metric on $P$ given by (2.2) and $\eta$ a connection form on $P$ such that $d \eta=\pi^{*} \Theta$. Let $\tau$ be a local cross-section of $P$ defined on a neighborhood $U$ of $x$ such that $\tau(x)=\tilde{x}$ and the differential map of $\tau$ maps the tangent space of $M$ at $x$ onto the horizontal space of $P$ at $\tilde{x}$, and $J_{\imath}$ the component of the almost complex structure $J$ with respect to the orthonormal frame field $\left\{X_{1}, \cdots, X_{n}\right\}$

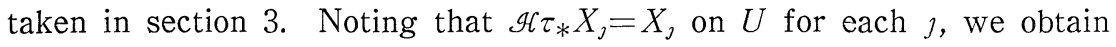

$$
\begin{aligned}
\Omega\left(\tilde{X}_{\jmath}, \tilde{X}_{k}\right)_{\tau(y)} & =\Omega\left(\tau_{*} X_{\jmath}, \tau_{*} X_{k}\right)_{\tau}(y)=\left(\tau^{*} \Omega\right)\left(\tilde{X}_{\jmath}, \tilde{X}_{k}\right) \\
& =-\left\langle J X_{\jmath}, X_{k}\right\rangle_{y}=-J_{j k},
\end{aligned}
$$

for any $y$ of $U$. Hence we see that

$$
\tilde{\nabla}_{\imath} \Omega_{j k}=\tilde{\nabla}_{\imath} \tilde{\nabla}_{j} \Omega_{k h}=0 .
$$

Now we define a linear connection $D$ on $P$,

$$
D_{\tilde{X}} \tilde{Y}=\tilde{\nabla}_{\tilde{X}} \tilde{Y}+T(\tilde{X}, \tilde{Y}) \text {, }
$$

for any vector fields $\tilde{X}, \tilde{Y}$ and $\tilde{Z}$. Then by (2.3) and (4.1), we have

$$
\left(D_{\tilde{X}} \tilde{g}\right)(\tilde{Y}, \tilde{Z})=0 \text {. }
$$

This means that $D$ is a metric connection. Since $M$ is locally symmetric Kählerian manifold, after a long calculation Lemmas 3.4, 4.1, 4.2, 4.3, 5.2 and 5.3 imply that for any vector field $\tilde{X}$, 


$$
D_{\tilde{X}} \tilde{R}=0 \quad \text { and } \quad D_{\tilde{X}} T=0 .
$$

By a result of W. Ambrose and I. M. Singer (see [1], p. 656), we find that $P$ is locally homogeneous. Thus we have

Proposition 5.1. Let $M$ be a locally symmetric Kählerian manifold and $P$ a principal circle bundle over $M$ with a Riemannian metric given by (2.2) and a connection form $\eta$ such that $d \eta=\pi^{*} \Theta$, where $\Theta$ is the fundamental 2-form of $M$. Then $P$ us locally homogeneous.

Furthermore we find from the definition of $T$ and (5.5) that

$$
\left(\tilde{\nabla}_{\tilde{X}} \tilde{R}\right)(\tilde{Y}, \tilde{X}) \tilde{X}=-T(\tilde{X}, \tilde{R}(\tilde{Y}, \tilde{X}) \tilde{X})+\tilde{R}(T(\tilde{X}, \tilde{Y}), \tilde{X}) \tilde{X}
$$

and

$$
\left(\tilde{\nabla}_{\tilde{X}} T\right)(\tilde{X}, \tilde{Y})=0,
$$

for any vector fields $\tilde{X}$ and $\tilde{Y}$. Thus by a result J. E. D'Atri and H. K. Nickerson (see [6], p. 252), we have

THEOREM 5.2. Let $M$ be a locally symmetric Kählerian manifold and $P$ a principal circle bundle over $M$ with a Riemannian metric given by (2.2) and a connection form $\eta$ such that $d \eta=\pi^{*} \Theta$. Then $P$ is a locally homogeneous and volume symmetric space.

Now let $\widetilde{R i c}$ and Ric be the Ricci tensor of $\tilde{g}$ and $g$ respectively. Then by Lemma 3.2 , we have the following

LEMMA 5.3 (cf. [10]).

(1) $\widetilde{\operatorname{Ric}}\left(\tilde{X}_{\imath}, \tilde{X}_{j}\right)=\operatorname{Ric}\left(X_{\imath}, X_{\jmath}\right)-2 t^{2} \delta_{i \jmath}$,

(2) $\widetilde{\operatorname{Ric}}\left(\tilde{X}_{\imath}, \xi\right)=0$,

(3) $\widetilde{\operatorname{Ric}}(\xi, \xi)=n t^{2}$.

By this lemma, S. Kobayashi [10] showed that if $M$ is Einsteinian, $\operatorname{Ric}\left(X_{\imath}, X_{j}\right)$ $=(S / n) \delta_{i j}$, then putting $t^{2}=S / n(n+2)$, we have an Einsteinian metric on $P$. Then he proved the following

Proposition 5.4. If $M$ is a complete Einstern-Kählerian manifold with positive scalar curvature, then we can construct a principal circle bundle $P$ over $M$ and specially an Einsterman metric with positive scalar curvature.

Combining Theorem 5.2 and Proposition 5.4, we have the following

THEOREM 5.5. If $M$ is a complete locally symmetric Einstein-Kählerian manifold with positive scalar curvature. Then we can construct a principal circle bundle $P$ over $M$, which is locally homogeneous and volume symmetric (especially even Einsternian). 
Now assume that the Einsteinian metric obtained in Proposition 5.4 satisfies the condition $(*)$. Then we first note that

$$
\Sigma \widetilde{R}_{o \alpha \beta \gamma} \tilde{R}_{o \alpha \beta \gamma}=2 \Sigma \widetilde{R}_{o \imath o j} \tilde{R}_{o \imath o j}=2 S^{2} / n(n+2)^{2},
$$

taking (1) of Lemma 3.2. Hence we have

$$
\begin{aligned}
\Sigma \widetilde{R}_{\alpha \beta \gamma \delta} \widetilde{R}_{\alpha \beta \gamma \delta} & =\Sigma \widetilde{R}_{o \beta \gamma \delta} \widetilde{R}_{o \beta \gamma \delta}+\sum \widetilde{R}_{\imath \beta \gamma \delta} \widetilde{R}_{i \beta \gamma \delta} \\
& =(n+1) \sum \widetilde{R}_{o \beta \gamma \delta} \widetilde{R}_{o \beta \gamma \delta}=2 \widetilde{S}^{2} / n(n+1) .
\end{aligned}
$$

This concludes that $P$ is of constant curvature. Thus we have

Proposition 5.6. A Riemannian metric on P obtained in Proposition 5.4 is an Einsternian manifold satisfying $(*)$ if and only of it is of constant curvature.

\section{An example.}

Consider the complex quadric of complex dimension 2, $Q^{2}(C)=C P^{1} \times C P^{1}$. Then $Q^{2}(C)$ admits the natural product Einstein-Kählerian metric, which is locally symmetric. Therefore by Theorem 5.5 , we can construct a principal circle bundle over $Q^{2}(C)$, which is homogeneous and volume symmetric (especially Einsteinian).

Now we give an explicit construction of this example. We take $G / H$ as follows :

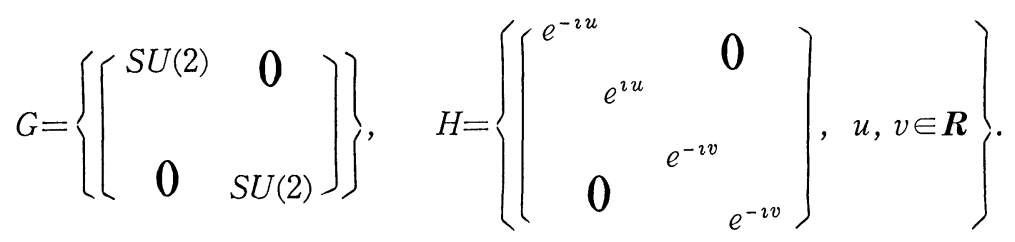

Then $G$ is a compact connected Lie group with the Lie algebra $g$ and $H$ a closed subgroup with the Lie algebra $\mathfrak{h}$. His locally the direct product of two closed normal subgroups $H_{1}$ and $H_{2}$ :

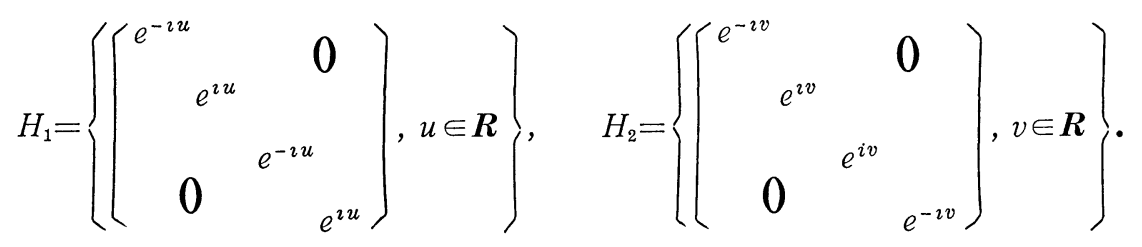

This means that $\mathfrak{h}=\mathfrak{h}_{1} \oplus \mathfrak{h}_{2}$ (direct sum of ideals), where $\mathfrak{h}_{1}$ and $\mathfrak{h}_{2}$ are the Lie algebras of $H_{1}$ and $H_{2}$, respectively. Then $P=G / H_{2}$ is a principal circle bundle over $G / H$ with projection $\pi$ and structure group $H_{1}$ where $\pi: P \rightarrow G / H$ is defined by $\pi\left(g H_{2}\right)=g H$ for $g \in G$ (see [9]). The $a d(G)$-invariant positive definite sym- 
metric bilinear form $B: \mathfrak{g} \times \mathfrak{g} \rightarrow R$ is taken to be $-(1 / 2)$ the killing form on $\mathfrak{g}=$ $s u(2)+s u(2)$. An orthonormal basis for $g$ with respect to this form is given by

$$
\begin{aligned}
& e_{1}=\left[\begin{array}{rrrr}
0 & 1 & 0 & 0 \\
-1 & 0 & 0 & 0 \\
0 & 0 & 0 & 0 \\
0 & 0 & 0 & 0
\end{array}\right], \quad e_{2}=\left[\begin{array}{llll}
0 & \imath & 0 & 0 \\
\imath & 0 & 0 & 0 \\
0 & 0 & 0 & 0 \\
0 & 0 & 0 & 0
\end{array}\right], \quad e_{3}=\left[\begin{array}{cccc}
0 & 0 & 0 & 0 \\
0 & 0 & 0 & 0 \\
0 & 0 & 0 & 1 \\
0 & 0 & -1 & 0
\end{array}\right], \\
& e_{4}=\left[\begin{array}{llll}
0 & 0 & 0 & 0 \\
0 & 0 & 0 & 0 \\
0 & 0 & 0 & i \\
0 & 0 & \imath & 0
\end{array}\right], \quad e_{5}=1 / \sqrt{2}\left[\begin{array}{cc}
-\imath & 0 \\
\imath & \\
0 & -\imath
\end{array}\right], \quad e_{6}=1 / \sqrt{2}\left[\begin{array}{cc}
-i & 0 \\
\imath & \\
0 & i
\end{array}\right] .
\end{aligned}
$$

Now note that $\left\{e_{5}, e_{6}\right\}$ is a basis for the Lie algebra $\mathfrak{h}$ of $H$, and $e_{6}$ a basis for the Lie algebra $\mathfrak{h}_{2}$. We set $\mathfrak{m}=$ the orthogonal complement of $\mathfrak{h}_{2}$ in $\mathfrak{g}$ spanned by $\left\{e_{1}, e_{2}, e_{3}, e_{4}, e_{5}\right\}$ and $\mathfrak{m}^{\prime}=$ the orthogonal complement of $\mathfrak{h}$ in $\mathfrak{g}$ spanned by $\left\{e_{1}, e_{2}, e_{3}, e_{4}\right\}$. Let $\langle$,$\rangle be the metric induced by restricting B$ to $\mathfrak{m}^{\prime},\langle,\rangle_{0^{\prime}}=B_{\mid \mathfrak{m}^{\prime}}$ and $J_{0^{\prime}}=-a d_{m^{\prime}}\left(e_{6}\right)$, where $\langle,\rangle_{0^{\prime}}\left(\right.$ resp. $\left.J_{o^{\prime}}\right)$ is the restriction of $\langle$,$\rangle (resp. J$ ) to the tangent space $T_{o^{\prime}}(G / H)$ of $G / H$ at $o^{\prime}=H$, identifying $\mathfrak{m}^{\prime}$ with $T_{o^{\prime}}(G / H)$. Then $(J,\langle\rangle$,$) is the natural (product) Kählerian structure on G / H$. Furthermore let $\widetilde{\langle,\rangle}$ be the metric induced by restricting $B$ to $\mathfrak{m}, \widetilde{\langle,\rangle_{0}}=B_{\mid \mathrm{m}}$, where $\widetilde{\langle,\rangle_{0}}$ is the restriction of $\widetilde{\langle,\rangle}$ to tangent space $T_{0}\left(G / H_{2}\right)$ of $G / H_{2}$ at $o=\left\{H_{2}\right\} \in G / H_{2}$, identifying $\mathfrak{n}$ with $T_{o}\left(G / H_{2}\right)$. Setting $\eta_{o}(X)=\left\langle X, e_{5}\right\rangle_{o}$ for $X \in \mathfrak{m}$, one can see that $\eta$ is a connection form on $G / H_{2}$ and $d \eta=\pi^{*} \Theta$ where $\Theta$ is the fundamental 2 -form of the Kählerian structure $(J,\langle\rangle$,$) .$

\section{BIBLIOGRAPHY}

[1] W. Ambrose and I.M. Singer, On homogeneous Riemannian manifolds, Duke Math. J., 25 (1958), 647-669.

[2] M. BERGER, Lew varietes riemanniennes homogenes normales simplement connexes a courbure strictement positive, Ann. Scuola Norm. Sup. Pisa, 15 (1961), $179-246$.

[3] A.L. BEsse, Manifolds all of whose geodesics are closed, Ergebnisse der Mathematik, vol. 93, Springer-Verlag, Berlin and New York, 1978.

[4] R.L. Bishop AND S.I. GoldberG, On the topology of positively curved Kaeler manifolds, Tôhoku Math. J., 15 (1963), 359-364.

[5] J.E. D'Atri AND H.K. Nickerson, Divergence-preserving geodesic symmetries, J. Differential Geometry, 3 (1969), 467-476.

[6] J.E. D'Atri and H.K. Nickerson, Geodesic symmetries in spaces with special curvature tensors, J. Differential Geometry, 9 (1974), 251-262.

[7] A. Gray, Pseudo-Riemannian almost product manifolds and submersion, J, Math. and Mech., 16 (1967), 715-738. 
[8] S. Ishihara And M. Konishi, Differential geometry of fibred space, Publications of the study group of geometry, vol. 9 (1973).

[9] G.R. JENSEN, Einsteın metrics on principal fibre bundles, J, Differential Geometry, 8 (1973), 599-614.

[10] S. KoBAYASHI, Topology of positively pinched Kaehler manifolds, Tôhoku Math. J., 15 (1963), 121-139.

[11] S. Kobayashi and K. Nomizu, Foundations of differential Geometry Interscience tract. Vol I, II, 1963, 1969.

[12] B. O'Neill, The fundamental equations of a submersion, Michigan Math. J., 13 (1966), 459-469.

[13] H.S. Ruse, A.G. Walker and T.J. Willmore, Harmonic Spaces, Edizionı Cremonesse, Roma, 1961.

[14] K. SaKamoto, On the topology of quaternion Kähler manifolds, Tôhoku Math. J., 26 (1974), 389-405.

[15] K. Sekigawa, Some Riemannian metrics on odd dimensional sphere, to appear.

[16] Y. Watanabe, Geodesic symmetries in Sasakian locally $\phi$-symmetric spaces, Kodai Math. J., 3 (1980), 48-55.

[17] Y. Watanabe, The curvature tensors of $S p(2) / S U(2)$ and $S U(5) / S p(2) \times S^{1}$ of Berger, to appear in Kodal Math. J., 5 (1982).

Department of Mathematics,

Faculty of Science,

TOYAMA UNIVERSITY

TOYAMA, JAPAN 